\title{
Women's views on autologous cell-based therapy for post-obstetric incontinence
}

\begin{abstract}
Aim: Fecal and urinary incontinence are devastating consequences of obstetricrelated perineal injury. The aim of the present study is to determine acceptability to parous women of autologous cell-based therapy for fecal and urinary incontinence that arises due to pelvic diaphragm tearing during vaginal childbirth. Materials \& methods: A multiple choice questionnaire survey was offered to 76 parous women at the Maternity Unit, University College Hospital, London, UK. Seventy completed questionnaires - response rate: $92 \%$. Results: In total, $84 \%$ of women indicated a willingness to accept autologous cell-based therapy for obstetric injury-induced incontinence rather than surgery. Conclusion: These observational data provide an indication of likely acceptance of autologous cell-based therapies for birth injury incontinence and will help with designing new therapeutic approaches.
\end{abstract}

First draft submitted: 10 August 2015; Accepted for publication: 18 December 2015; Published online: 15 February 2016

Keywords: anal sphincter $\bullet$ autologous stem cell therapy $\bullet$ fecal incontinence $\bullet$ obstetric injury $\bullet$ patient preference $\bullet$ urinary incontinence

Fecal incontinence ([FI] urge and accidental bowel leakage) and stress urinary incontinence (SUI) are distressing and life-limiting conditions that affect women after childbirth. Obstetric trauma to muscles, nerves, or connective tissues of the pelvic diaphragm is the commonest cause of FI [1] and occurs in $10-15 \%$ of vaginal deliveries $[2,3]$. Therapeutic strategies to address these symptoms are generally not curative - surgical repair is associated with poor long-term outcomes [4-6] and conservative therapies are primarily aimed at palliation. The majority of those patients will develop incontinence symptoms many years after childbirth. Recent preclinical and early-stage clinical studies have indicated cell-based strategies using autologous cells offer the potential to regenerate damaged urethral $[7,8]$ and anal [9] sphincter muscle to cure SUI and FI.

Compounding this problem, parous women who present with pelvic floor tears and prolapse sustained during childbirth have a poor understanding of long-term healthcare options for obstetric injury risk. It is unclear to what extent women would be prepared to undertake specialist assessment and cell-based treatment for symptoms of SUI and FI. Such information is fundamental to the design and implementation of studies investigating the development of cell-based therapies. The primary objective of the current study is to establish the opinions of parous women for autologous cell-based therapy that may provide prophylactic and curative solutions for FI and SUI.

A diverse range of therapeutic interventions that are selected based upon the severity of FI or SUI are currently available. These include biofeedback (e.g., pelvic floor exercises) [10,11], surgery to repair sphincters involving muscle transfer procedures [12], transplantation of artificial sphincters [13-15]
Bernice Wright ${ }^{1}$, Anton Emmanuel $^{2}$, Eleni Athanasakos ${ }^{2}$, Nina Parmar', Georgina Parker ${ }^{3}$, Belinda Green ${ }^{3}$, Emma Tailby ${ }^{3}$, Heidi Chandler ${ }^{3}$, Julyette Cushnie ${ }^{3}$, June Pembroke ${ }^{3}$, Yvonne Saruchera ${ }^{3}$, Arvind Vashisht ${ }^{3}$ \& Richard Day*,1

${ }^{1}$ Applied Biomedical Engineering Group, University College London, Rockefeller Building, 21 University Street, London, WC1E 6JJ, UK

${ }^{2}$ GI Physiology Unit, University College Hospital, 235 Euston Road, London, NW1 2BU, UK

${ }^{3}$ University College Hospital, Elizabeth Garrett Anderson Wing, Institute for Women's Health, London, WC1E 6BD, UK

*Author for correspondence: r.m.day@ucl.ac.uk 
or muscle with stimulating electrodes [16,17], sacral nerve stimulation [18-20], vaginal slings [21,22], and/or recommendation of elective caesarean sections for future pregnancies [23] in high-risk cases (due to previous traumatic damage to the pelvic floor). Intractable SUI and FI are typically managed with catheter placement and permanent colostomy, respectively. Thorough pelvic examinations [24] following vaginal deliveries can aid early diagnosis of risk of SUI and FI.

Many of the therapies currently being developed for SUI and FI are designed to offer curative options that can supersede existing clinical interventions that may only allow a measure of symptom control. In the present study, an emerging cell-based therapy consisting of autologous skeletal myoblasts attached to biodegradable microspheres was explained to parous women as a potential curative treatment option for SUI and FI.

The premise for the present research is supported by a study demonstrating the technical feasibility of delivering autologous skeletal myoblasts as a potential treatment for FI [9]. In that uncontrolled pilot study, cells harvested from a pectoralis muscle biopsy were injected into the external anal sphincter defect using direct ultrasound guidance. Data at 1 year indicated a decrease in the Wexner score and patients reported improved quality of life but anorectal physiology (anal squeeze and resting pressure) was not improved. A recent 5-year follow-up observational study [25] of the same group suggests a general increase in anal squeeze and resting pressure at 5 years compared with baseline. While this remains an uncontrolled study it indicates the potential benefit of cell-based therapy for FI.

In the present study we developed a multiple choice questionnaire to assess whether or not primiparous and multiparous women would consider complex specialist evaluation and treatment following perineal birth trauma. The purpose of the questionnaire was as a tool to provide an initial observation of women's opinions on cell-based incontinence therapy. The use of biomaterials to deliver autologous cells to treat FI and SUI was described in lay terms to parous women using diagrams. Data derived from the observational questionnaire will help with the design of new therapeutic strategies as well as forming the basis for more in-depth questionnaires specifically outlining the detail of cell-based therapies for birth injury-related FI and SUI. Here, we examined whether women's beliefs/views to undergo enhanced evaluation and cell-based therapy related to the presence of symptoms of incontinence. The key finding in this observational study revealed that the majority of women were willing to accept an autologous cell-based therapy for obstetric injuryinduced incontinence. This finding correlated with worry about developing FI and SUI later in life as well as acceptance for assessment of obstetric risk. We therefore concluded that from the stand-point of parous women, there is a strong case for development of autologous cell-based therapeutic interventions as prophylactic or treatment measures against obstetric-injury related FI and SUI.

\section{Patients \& methods}

\section{Patients \& study design}

A questionnaire survey of women ( $\mathrm{n}=76$ with $\mathrm{n}=70$ respondents) attending the Maternity Unit at University College Hospital (London, UK) for postnatal care was undertaken. All women who provided informed consent to participate had previously had children in the unit and the cohort included a mixed group with various degrees of obstetric injuries. To be eligible for the study, it was necessary for the women involved to have had a vaginal delivery and be between the ages of 16 and 50 years. Participants were approached and invited to complete a questionnaire structured to determine their views and preferences with regard to specialist assessment and treatment with an autologous cell-based therapy to treat incontinence. The women verbally agreed to this questionnaire-based participation. We did not obtain informed formal consent as this was a survey questionnaire to obtain opinions unrelated to individual therapy decisions. The multiple choice questionnaire is shown as Supplementary Data.

\section{Questionnaire analysis}

The multiple choice questionnaire (see Supplementary Data) was presented to patients with explanations (delivered verbally by the interviewer) of the cell-based therapy aimed at treating obstetric-injury induced SUI and FI. The questionnaire was structured as a series of questions that established the age range, parity, continence, willingness to opt for assessment of obstetric risk, feelings about developing SUI and FI later in life, willingness to consider cell-based therapy for incontinence, thoughts regarding timing of treatment, and questions and comments from women about the novel therapy. Correlations between responses, for example, willingness to be assessed for obstetric risk and continence were analyzed. This was primarily an observational study. The responses to the query for willingness to opt for cell-based therapy were interrogated with follow-up questions involving a scoring scale of 1-10. We classified responses 7-10 as "willing to be treated," responses 4-6 as "unsure about treatment," responses $0-3$ as "unwilling to 
have treatment." Statistical significance between data trends was determined using SPSS (version 22 for Windows. Armonk NY, USA).

\section{Results}

\section{Demographics}

The modal age group was $26-35$ years $(49 \%$; 34 out of 70 ), with $40 \%$ (28 out of 70 ) aged $36-45$ years, $7 \%$ (five out of 70) aged 20-25 years old, and 4\% (three out of 70$)$ aged over 46 years. The majority of women were uniparous $(67 \%$; 47 out of 70 ) and $33 \%$ (23 out of 70) were multiparous. Within those groups, multiparous women in the 20-25 years and 26-35 years subgroups had less than four children. The majority of multiparous women in the 36-45 years age range had two children and those who were more than 46 years old tended to have more than two children.

\section{Symptoms}

In total, $21 \%$ (15 out of 70) of the cohort were sometimes faecally incontinent at the time of completion of the questionnaire and 7\% presented with fecal incontinence (five out of 70). The ability of women to control their bowels did not correlate with their age, their parity, or the period of time after deliveries.

\section{Willingness to be assessed for obstetric risk}

The majority of the cohort $(90 \%$; 63 out of 70$)$ expressed a desire to be assessed for risk of obstetric injury before giving birth (Figure 1). This was independent of their current ability to control their bow- els. One woman out of $63(2 \%)$ within the group that accepted assessment of obstetric risk was incontinent, 13 out $63(21 \%)$ were sometimes incontinent, and remaining 49 (out of 63: 78\%) were continent. Only two women (out of 70) did not want to be assessed for obstetric risk. There was no significant relationship $(p=0.26)$ between willingness to be assessed for obstetric risk and continence.

\section{Feelings about developing stress urinary \& fecal incontinence later in life}

A greater percentage of the cohort were worried about developing SUI and FI later in life (80\%; 56 out of 70) than those who were unsure $(6 \%$; four out of 70$)$ or not worried (14\%; ten out of 70 ) (Figure 2). Within the group of women who were worried, 14 women (14 out of 56; 25\%) were sometimes unable to control bowels, five (five out of 56; 9\%) were incontinent, and the remaining women (37 out of 56) were continent (Figure 2). There was a significant correlation ( $\mathrm{p}=0.0001)$ between current symptoms of incontinence and worry about developing SUI and FI later in life.

\section{Willingness to accept cell-based therapy for incontinence}

In total, $79 \%$ ( 55 out of 70 ) of the cohort were willing to opt for cell-based therapy to reduce incontinence later in life (Figure 3A). The majority of this group (49 out of 55) was worried about developing SUI and FI later in life. Only two women (out of 70) - one of whom was not worried about developing inconti-

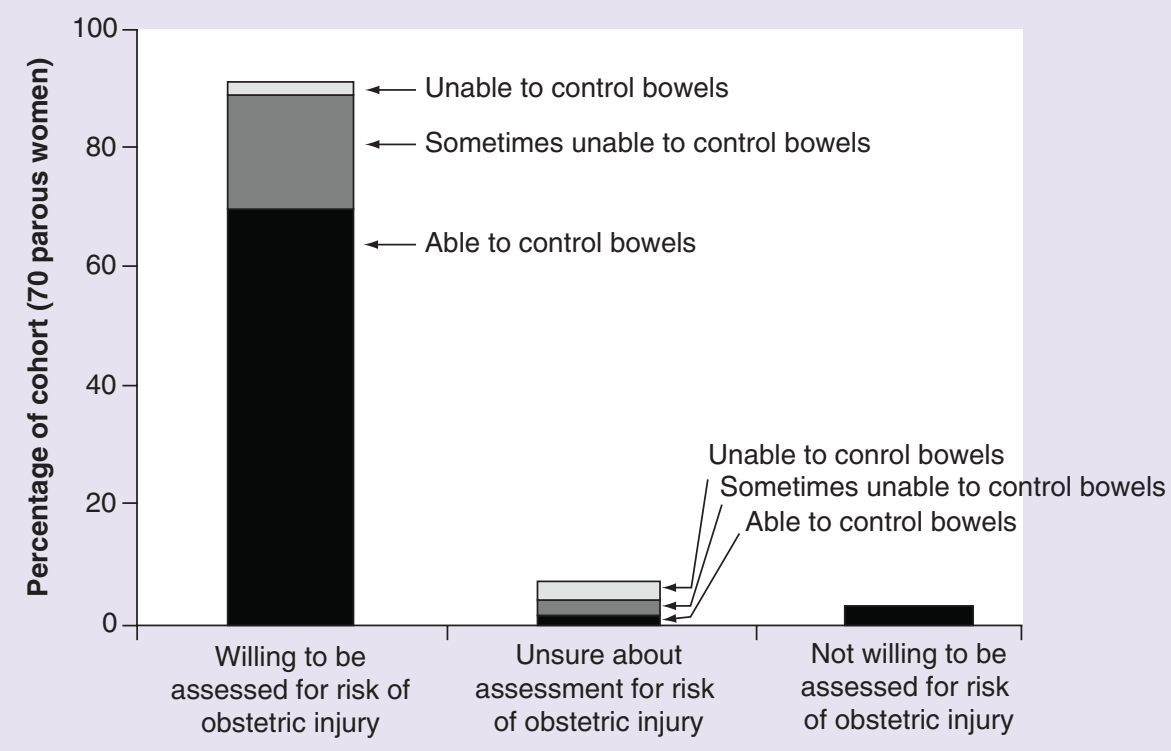

Figure 1. Correlations between willingness to be assessed for obstetric risk correlated and incontinence.

Assessments for obstetric risk were acceptable for women whether or not they presented fecal incontinence. Percentages are a proportion of the cohort consisting of 70 parous women. 


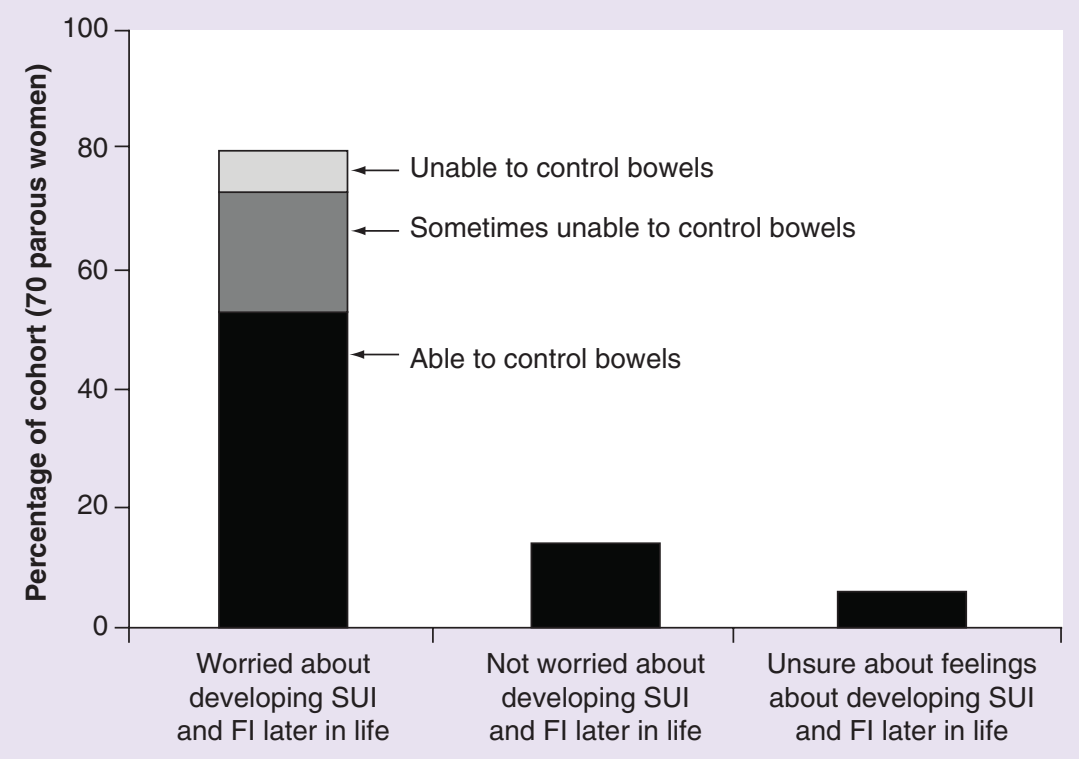

Figure 2. Parous women were worried about developing incontinence later in life. Women were worried about developing SUI and FI later in life regardless of ability to control bowels. Percentages are a proportion of the cohort consisting of 70 parous women.

$\mathrm{FI}$ : Fecal incontinence; SUI: Stress urinary incontinence.

nence - indicated that they would not opt for a novel cell-based therapy. In total, $84 \%$ (59 out of 70 ) of the cohort indicated willingness for cell therapy over symptom control options and $1 \%$ (one out of 70 ) were unwilling, with $14 \%$ (ten out of 70 ) unsure (Figure 3B).

A further details of cell therapy explored was with regard to having autologous cells attached to biodegradable microspheres placed in the urethral and anal sphincters. A slightly lower proportion of women $(71 \%$; 50 out of 70) were willing to consider such treatment as an alternative to a surgical operation, $23 \%$ (16 out of 70 ) were unsure and $4 \%$ (three out of 70 ) would not consider it, and $1 \%$ (one out of 70) may or may not consider autologous cell-based therapy instead of a surgical operation (Figure $3 \mathrm{C}$ ). There was a significant correlation $(p=0.0002)$ between worry of developing incontinence later in life and willingness to consider autologous cell-based therapy. Willingness to accept autologous cell-based therapy over symptom control correlated significantly $(\mathrm{p}=0.0001)$ with worry about developing SUI and FI. Lack of acceptance of autologous cell-based therapy in the absence of incontinence symptoms was also significant $(\mathrm{p}=0.0001)$. Taken together, these data demonstrated that sequential, follow-up inquiry potentially highlighted a distinct subset that was willing to adopt a novel autologous cell-based therapy for SUI and FI.

More than half of the cohort $(51 \%$; 36 out of 70$)$ were willing to opt for treatment in the absence of SUI and FI symptoms. However, 37\% (26 out of 70) were unsure, and $11 \%$ (eight out of 70) would not opt for novel treatments in the absence of SUI and FI symptoms (Figure 4A). By contrast, when women were asked if they would accept treatment if presenting with symptoms of FI (Figure 4B), the majority (89\%; 62 out of 70) scored yes and only one patient indicated that she would not like the novel therapy in such circumstances. Questions relating to administration of the cell-based therapy correlated well to responses for willingness to accept novel therapy for SUI and FI. The majority of women who would consent to a muscle biopsy for collection of muscle cells (Figure 5A) for the novel treatment $(61 \%$; 43 out of 70$)$, also indicated that they would opt for autologous cell therapy for SUI and FI (91\%; 39 out of 43). Approximately one third of the cohort $(31 \% ; 22$ out of 70$)$ were unsure about allowing a muscle biopsy, but within that group, $45 \%$ (ten out 22) had previously been unsure about opting for the novel therapy over symptom control. There were significant levels $(p=0.00009)$ of uncertainty within the cohort for allowing a muscle biopsy. In total, $64 \%$ (45 out of 70) of parous women would participate in a clinical trial and $30 \%$ (21 out of 70 ) were unsure (Figure 5B).

\section{Timing of treatment}

The most popular preference for when to initiate treatment was immediately after childbirth (31\%: 22 out of 70) (Figure 6). A subgroup of 18 women (26\%; 18 out of 70) would opt for therapy before childbirth, 
(A)
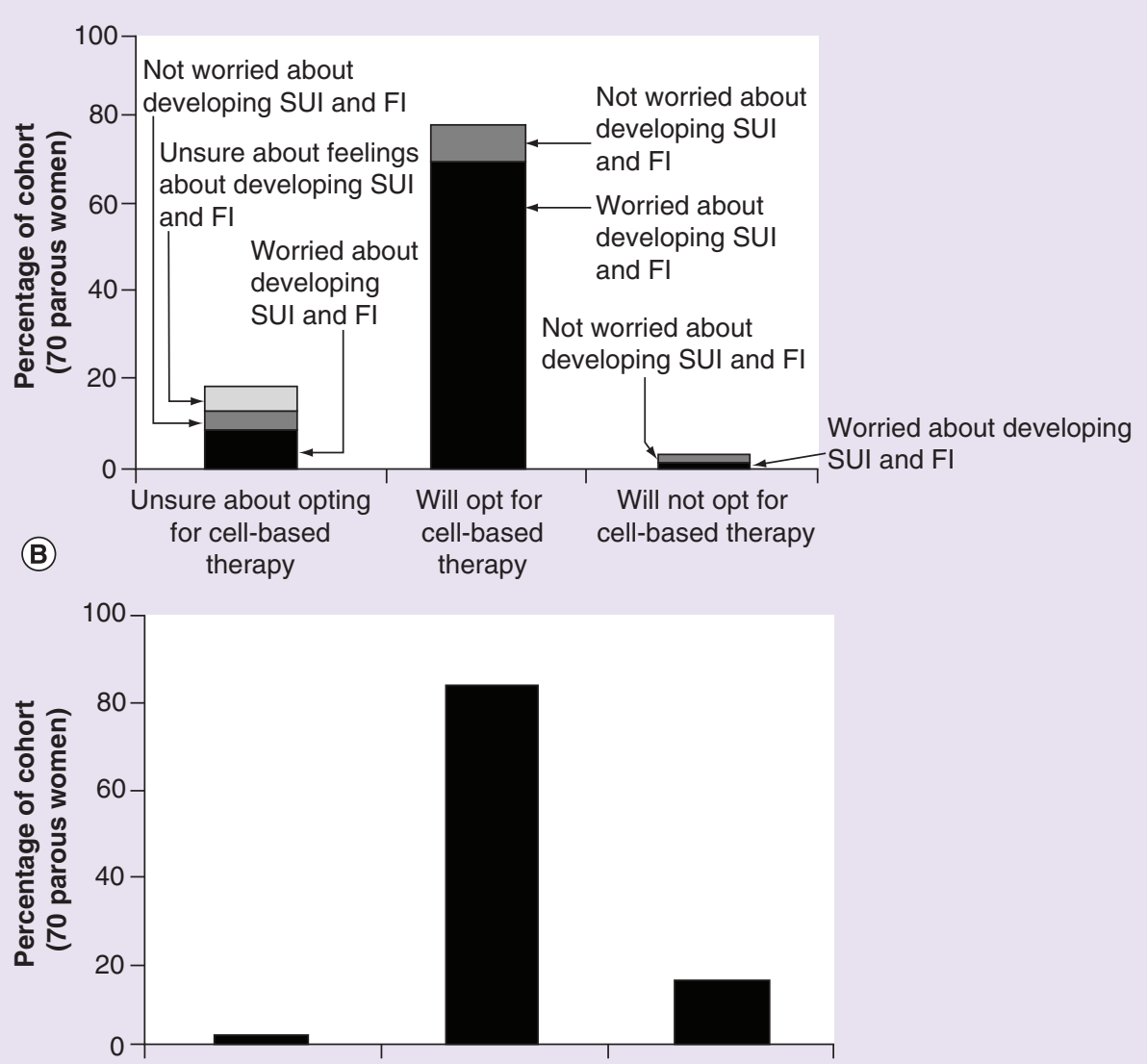

Not willing to opt Willing to opt for Unsure about opting for cell-based cell-based for cell-based therapy over therapy over therapy over
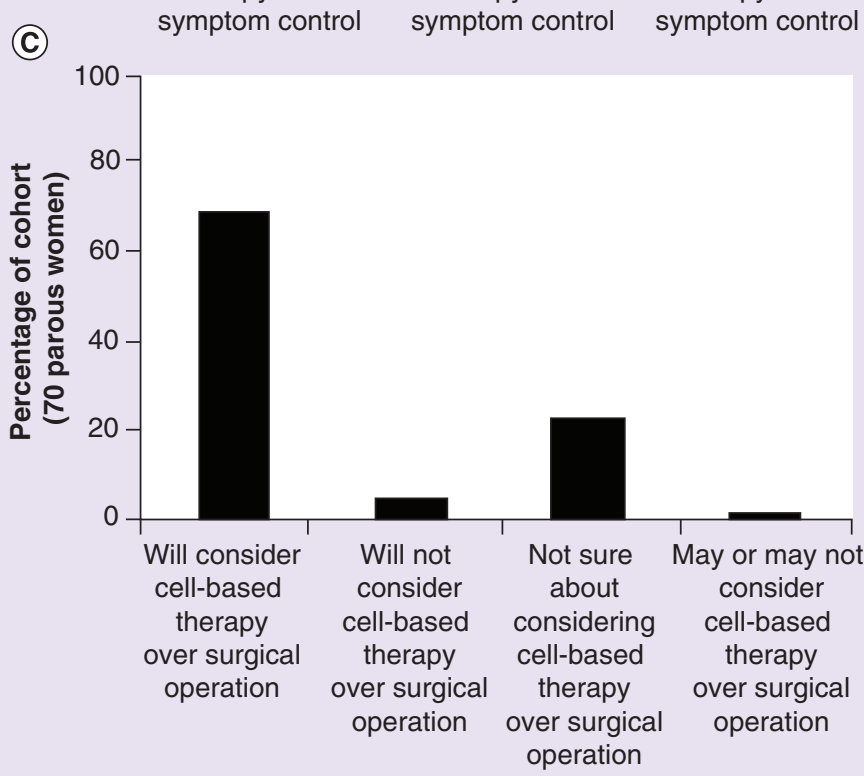

Figure 3. Parous women accept a cell-based therapy for incontinence. The majority of women who were willing to opt for cell-based therapy for SUI and FI were worried about developing incontinence later in life (A). The greatest proportion of the cohort $(n=70)$ were willing to opt for the therapy as an alternative to symptom control (B) or a surgical operation (C). Percentages are a proportion of the cohort consisting of 70 parous women.

FI: Fecal incontinence; SUI: Stress urinary incontinence. 
(A)

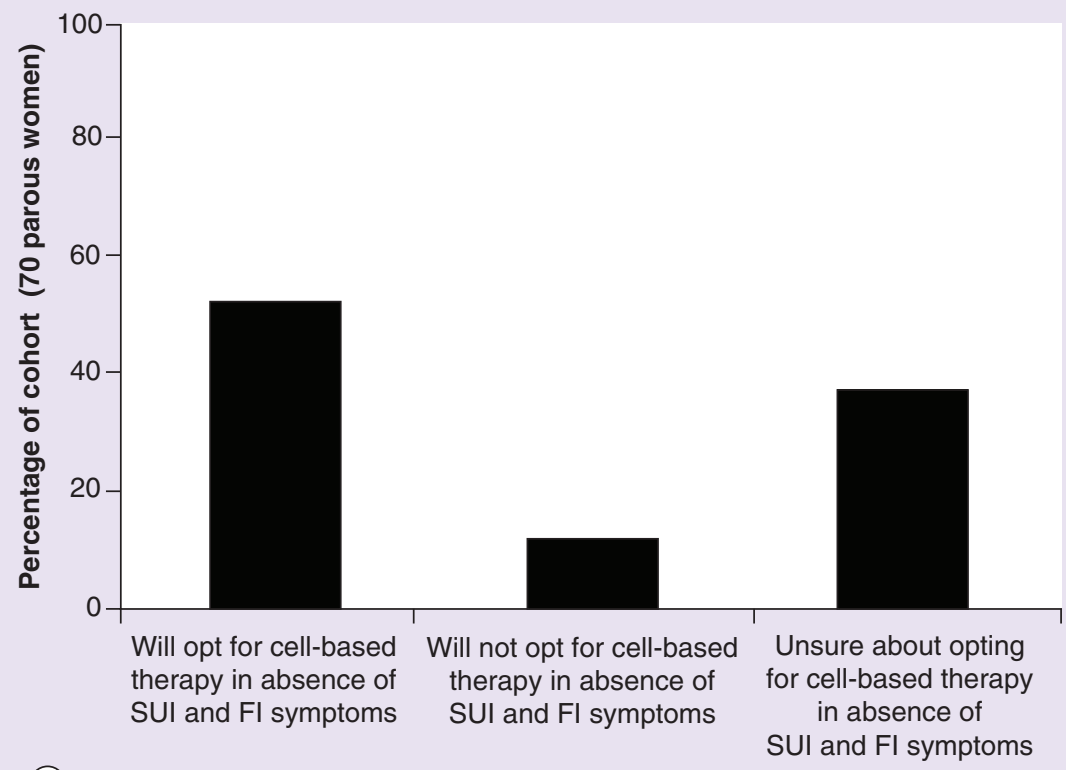

(B)

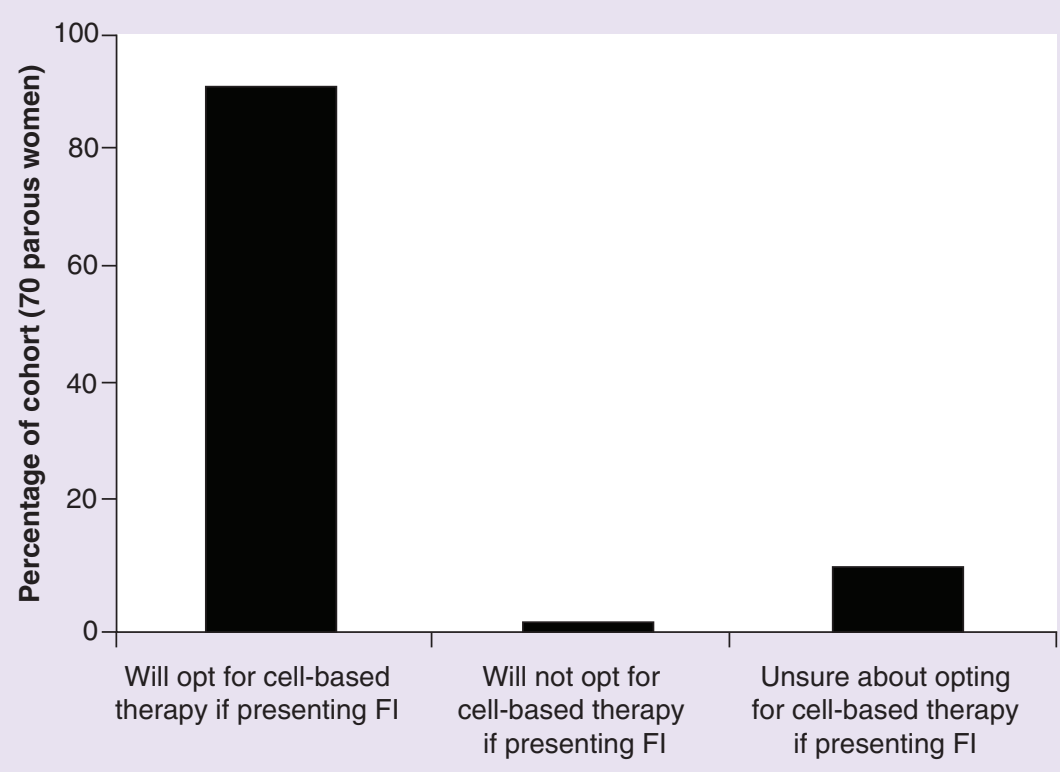

Figure 4. Acceptance of the cell-based therapy in the absence of incontinence symptoms was less avid than in the presence of those symptoms. The percentage of parous women who were willing to accept cell-based therapy in the absence of SUI and FI symptoms was similar to the proportion who was unsure (A). The majority of the cohort accepted the cell therapy in the presence of Fl symptoms (B). Percentages are a proportion of the cohort consisting of 70 parous women.

FI: Fecal incontinence; SUI: Stress urinary incontinence.

$20 \%$ of the cohort (14 out of 70 ) preferred treatment 6 months after childbirth. The least popular preference was for initiation of treatment more than 6 months after childbirth (9\%; six out of 70$)$. A proportion of the cohort (10\%; eight out of 70 ) were partial to multiple treatment periods, for example, before, immediately after, 6 months after, and more than 6 months after childbirth or before and immediately after childbirth. There were no convincing trends between willingness to opt for the novel therapy or feelings about developing SUI and FI later in life and individual options for when to initiate therapy. 
Questions \& comments from women about the cell-based therapy

The questionnaire included a free text option and the majority of questions or comments related to risks involved with the novel therapy. Less frequently there were questions about the efficacy of the therapy and least often asked were practical questions about the practicalities of timings of treatment and recovery. There were individual comments about ethics of cell therapy and the need for prevention rather than cure of traumatic obstetric injuries.

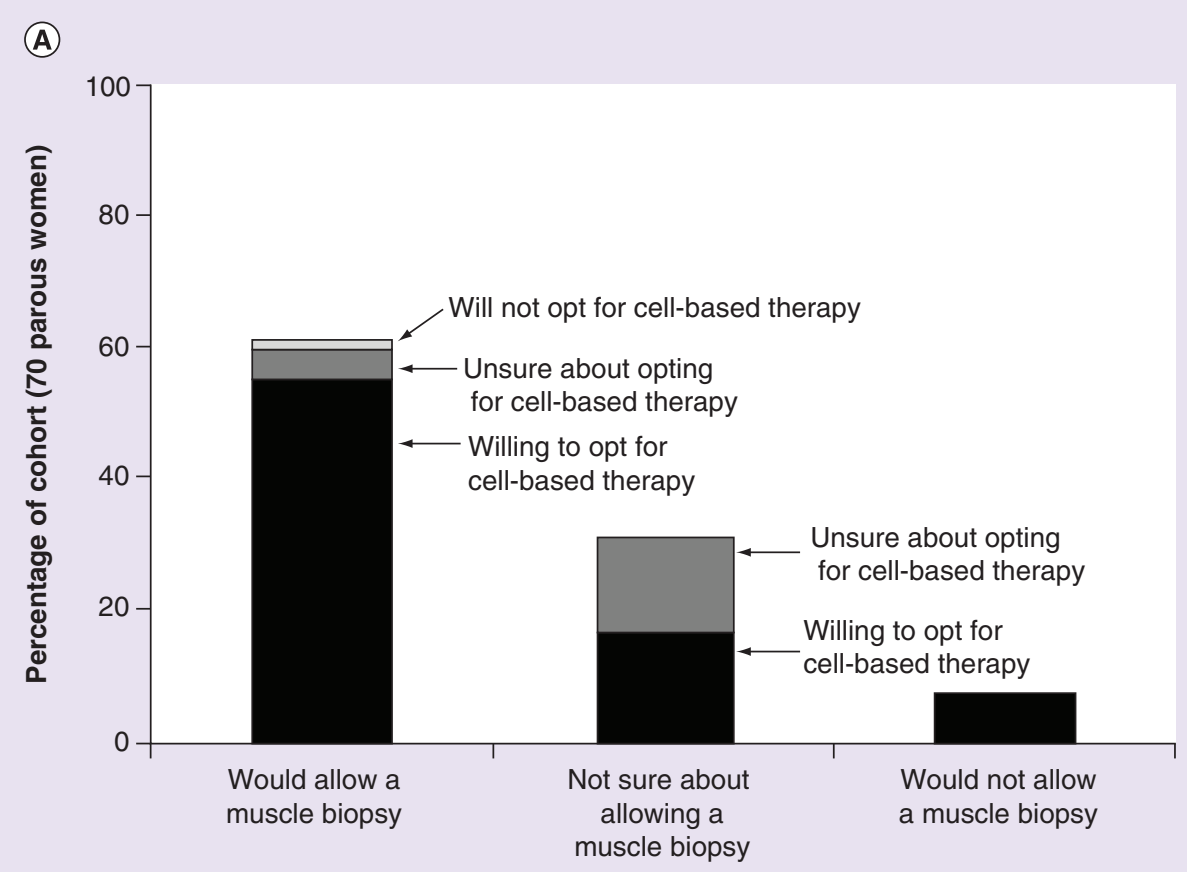

(B)

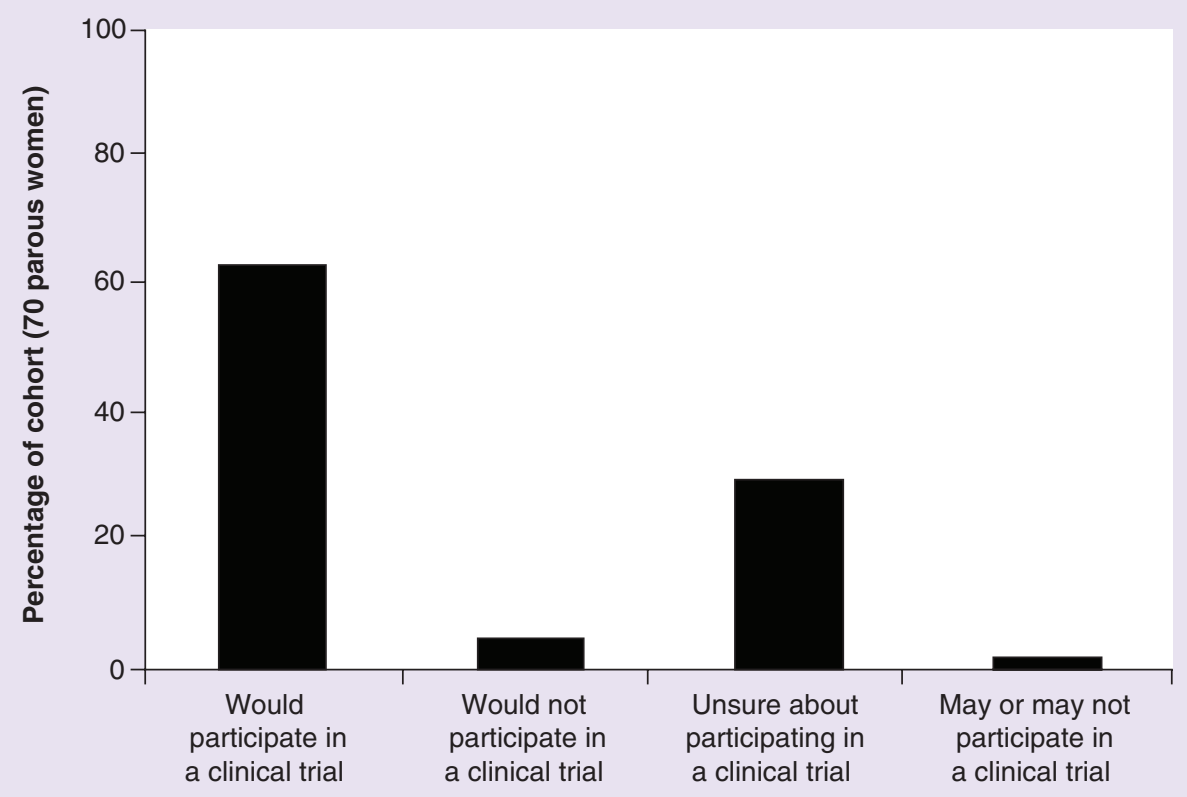

Figure 5. Parous women were willing to contribute autologous muscle cells for cell-base therapy and participate in related clinical trials. The majority of the cohort was willing to contribute a muscle biopsy for harvesting autologous cells (A) and they were also willing to participate in a clinical trial of this type of therapy (B). Percentages are a proportion of the cohort consisting of 70 parous women. 


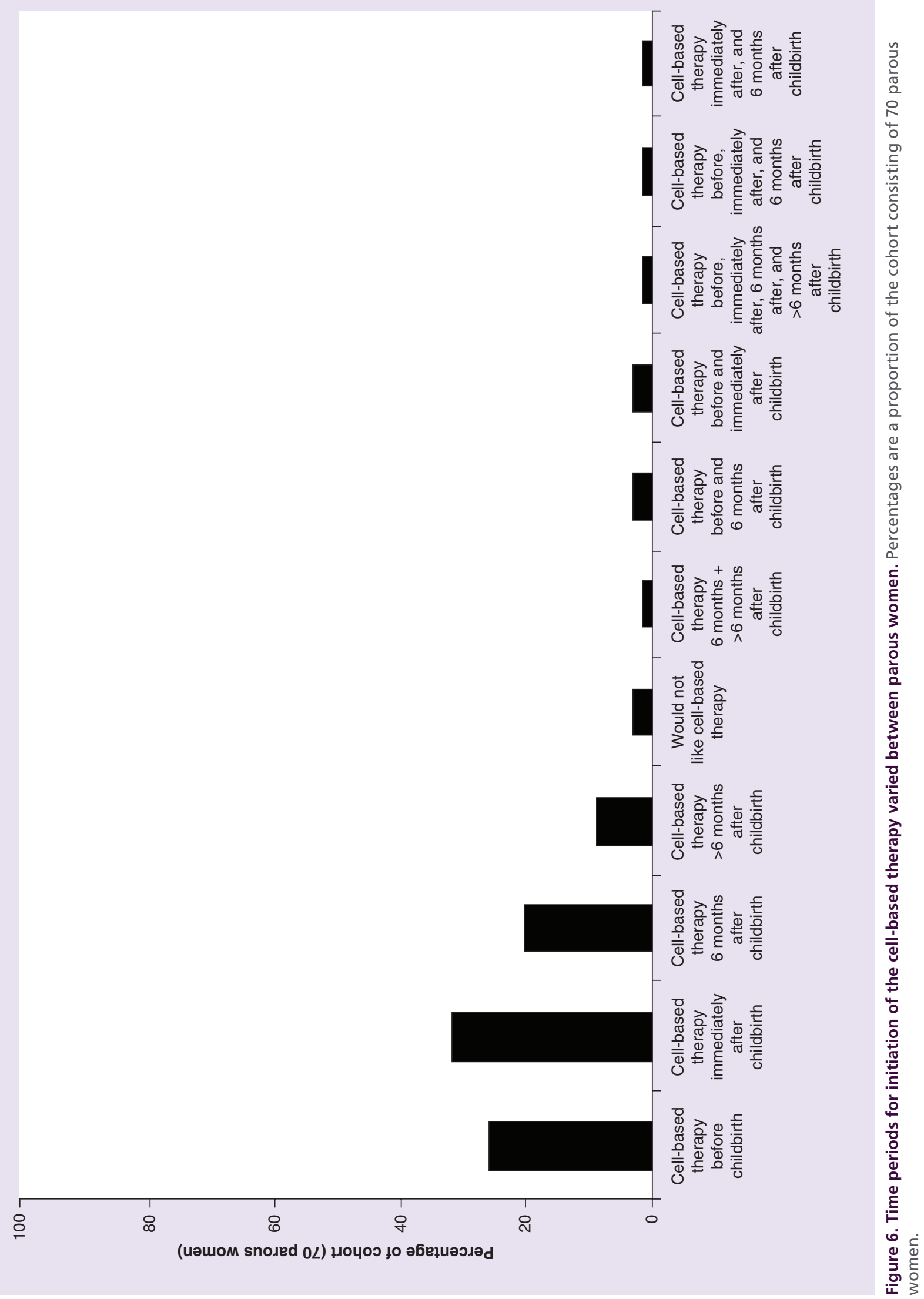




\section{Discussion}

Principal findings

Acceptance levels were high, with four out of every five women (from the cohort of 70 women) amenable to an autologous cell-based therapeutic modality as a potentially curative treatment for obstetric injuryinduced SUI and FI. This strong trend in approval for a cell-based therapy as opposed to surgery, correlated with feelings of worry $(83 \% ; 49$ out of 59 of women were worried about developing SUI or FI later in life) of women as well as willingness to consider the novel therapy over surgery (71\%: 50 out of 70). This subgroup of women was also worried about developing SUI and FI later in life. Similarly, willingness to undergo assessment for obstetric injury risk was a prominent response ( $88 \%$ were willing) that could be associated with agreement for the autologous cellbased therapy. These data suggest that fear of latent development of SUI and FI is an important underlying factor in deciding to undergo specialist assessment and complex therapy for these symptoms.

Uncertainty about muscle biopsy may be due to fear of pain, scarring, infections, and long-term impairment of muscle functions following removal of the tissue. At present, information about the size of the muscle biopsy required for collection of cells, protocols for acquiring the biopsy, and follow-up care to minimize scarring and trauma to the harvest site are evolving. The results of this questionnaire study suggest that revealing details about the requirements for receiving the cell-based therapy may distinguish groups who are genuinely interested in its clinical application from those who may only support the concept of a curative therapy for SUI and FI.

Individual options for when to initiate therapy were not influenced by willingness to opt for the novel therapy or feelings about developing SUI and FI later in life. These data indicate that questions related to when to initiate treatment may be abstract at this early preclinical stage and need a clearer evidence base to help women indicate firm preferences for timing of therapy.

\section{Strengths \& limitations}

The main strength of the present study was the clear correlation between acceptability of the autologous cell-based therapy and worry about developing FI and SUI later in life. These data provide a basis from which patients' compliance for future therapies can be anticipated and quantified. The associated limitation, however, was the lack of a strong evidence base describing the application and clinical benefits of the cell-based therapy. This drawback was reflected in questions and comments that were put forward by a number of women who participated in the survey. This limitation will be addressed with ongoing clinical and preclinical studies from our group and other groups that involve testing the efficacy of different cell-based therapeutic strategies. A key asset of this study is that women indicated little awareness of assessment for obstetric risk factors - the majority signposted their interest in receiving antenatal evaluation for risk of obstetric injury. This response may lead to further studies investigating current practice in ante- and post-natal care for women in the UK. Increasing the size of the cohort would allow a more reliable estimation of the primary concerns of parous women with regard to reproductive health, as well as allow better study of correlation between treatment acceptance and age, parity, and current bowel/bladder control.

\section{Comparison with existing literature}

Studies investigating patients' views on prospective therapies for obstetric injury dependent stress urinary and fecal incontinence are scarce. Cohort studies describing treatment preferences for urinary incontinence have previously been reported [26-28]. Those, however, present therapeutic modalities that are validated and clinically available. One study elicited preferences for urinary continence treatment for residents in long-term care from their proxy care providers [27]. The preferred treatment options were the noninvasive strategies, prompted voiding and diapers rather than the invasive strategies, indwelling catheters and electrical stimulation. Older adults preferred therapies directed at the underlying causes of urinary incontinence, in other words, drug medication and electrical stimulation. That survey is comparable to the main section of the present study concerned with establishing women's views on a cell-based therapy as opposed to conventional surgical procedures. In the current study there was no correlation between age and treatment preference - the majority of parous women in the 20-25-year, 26-35-year, and 36-45-year age ranges chose the option of a cell-based therapy rather than surgery.

The lack of awareness of procedures for assessment of obstetric risk and the impact of obstetric injuries encountered in the present study highlights a critical need in women's reproductive health provision in the UK. A previous study [26] described a similar issue in Boston (MA, USA) within a community of black, Hispanic, and white adults. More than $40 \%$ of women in the cohort (5506 adults) and approximately $20 \%$ of men with weekly incontinence reported that they had never sought care for this condition. From those who pursued treatment, $60 \%$ received it, but half of those continued to experience daily leakage. A conclusion of the study was that the majority of incontinent adults in the cohort who sought treatment did not believe that 
it was provided as symptoms continued. Apathy from healthcare providers with regard to efficacy of therapies was cited as an underlying cause for unsatisfactory therapy for urinary incontinence.

\section{Implications for clinicians \& policy-makers}

This study has drawn together a number of distinct strands of inquiry concerning women's acceptance of therapy for SUI and FI. Responses indicated the importance of raising awareness of obstetric injury risk and the occurrence of incontinence due to those injuries. Procedures to integrate screening procedures for assessment of obstetric risk, treatment strategies for prospective obstetric injuries, and measures for prevention of obstetric injuries into current antenatal care programs is a challenge for hard-pressed services.

\section{Unanswered questions \& future research}

Future studies of invasive incontinence therapies should include detailed information on the administration of the new types of therapy and assessment of patients' willingness to undergo such therapies. Separate studies to investigate knowledge gaps in populations of parous women regarding existing and possible options at both ante- and post-natal stages would also be informative.

\section{Conclusion}

The present study demonstrates a clear trend in acceptance for autologous cell-based therapy for SUI and FI. The correlation of fear of developing SUI and FI and general agreement to receive assessment for risk of obstetric injury with compliance for a novel therapy indicates the importance of addressing this issue honestly and empathically. The clinical requi- site for enhanced therapy to reduce the incidence of late-onset SUI and FI that arise due to obstetric injuries is stressed. Furthermore, addressing this aspect of human reproductive morbidity will allow reforms in the quality and consistency of clinical and physiological care provided to parous women. Regenerative and/or prophylactic therapy for obstetric injuries can complement campaigns to raise awareness of ante- and postnatal care that may in turn destigmatize these life-altering symptoms. Prospective studies that follow-on from the work described here will involve more in-depth evaluations of women's views on autologous cell-based therapy once they have been provided with more information regarding this treatment option.

\section{Future perspective}

The evolution of therapy for obstetric-related FI and SUI is critically dependent on accurate assessment and quantification of patient needs in parallel with development of therapies. Over the next 5-10 years, it is likely that new therapeutic strategies based on autologous skeletal myoblasts alone or attached to biodegradable materials will advance to human clinical trials. The evidence base for ethical approval of those trials will include validated questionnaires from parous women responding to the likely risk/benefit based on the outcome of preclinical studies. Furthermore, standardized clinical assessment of women's risk for obstetric injuries will inform this clinical work and drive development of pre-emptive strategies for managing perineal tears. Therapeutic tools such as those outlined in the current study may allow women greater control and longevity in their reproductive life span and health.

Executive summary

Need for women's opinions on pre-emptive autologous cell therapy for fecal \& stress urinary

incontinence due to childbirth injuries

- Parous women who present with pelvic floor tears and prolapse sustained during childbirth have a poor understanding of long-term healthcare options for obstetric injury risk.

- Addressing the underlying cause of fecal incontinence (FI) and stress urinary incontinence (SUI) at an early stage, before symptoms have emerged, may be key to preventing onset later in life. Women's perception of this type of pre-emptive approach can accelerate development of therapies for FI and SUI.

Response of parous women to novel, developing autologous cell therapy for FI \& SUI

- A multiple choice questionnaire was developed to assess whether or not primiparous and multiparous women would consider complex specialist evaluation and treatment following perineal birth trauma.

- Beliefs/views to undergo enhanced evaluation and cell-based therapy related to the presence of symptoms of incontinence were also investigated.

- The majority of women were willing to accept an autologous cell-based therapy for obstetric injury induced incontinence.

Usefulness of acquiring women's views on developing therapies for FI \& SUI

- Addressing the impact of perineal injury will allow reforms in the quality and consistency of clinical and physiological care provided to parous women.

- Regenerative and/or prophylactic therapy for obstetric injuries can complement campaigns to raise awareness of ante- and post-natal care that may in turn destigmatize these life-altering symptoms. 


\section{Supplementary data}

To view the supplementary data that accompany this paper please visit the journal website at: www.futuremedicine.com/ doi/full/10.2217/rme.15.88

\section{Financial \& competing interests disclosure}

The project was supported by grants from the UK Medical Research Council (MR/L002752/1), The Sir Halley Stewart Trust, The Henry Smith Charity, and the UCL Grand Challenge Studentship Scheme. The research was undertaken at UCL/UCLH which receives funding from the Department of Health's NIHR as a Comprehensive Biomedical Research

\section{References}

Papers of special note have been highlighted as:

- of interest; $\bullet \bullet$ of considerable interest

1 Dudding TC, Vaizey CJ, Kamm MA. Obstetric anal sphincter injury: incidence, risk factors, and management. Ann. Surg. 247(2), 224-237 (2008).

2 Evers EC, Blomquist JL, McDermott KC et al. Obstetrical anal sphincter laceration and anal incontinence $5-10$ years after childbirth. Am. J. Obstet. Gynecol. 207(5), e1-e6 (2012).

- Anal sphincter laceration that occurs in women during their first child birth delivery is associated with fecal incontinence after $5-10$ years.

3 Samarasekera DN, Bekhit MT, Wright Y et al. Long-term anal continence and quality of life following postpartum anal sphincter injury. Col. Dis. 10(8), 793-799 (2008).

- Primary surgical repair of pelvic floor tearing due to vaginal childbirth does not support long-term anorectal function. Prevention and minimization of further damage to the pelvic diaphragm in subsequent deliveries is important to prevent further sphincter damage.

4 Halverson AL, Hull TL. Long-term outcome of overlapping anal sphincter repair. Dis. Colon Rectum 45(3), 345-348 (2002).

5 Malouf AJ, Norton CS, Engel AF et al. Long-term results of overlapping anterior anal-sphincter repair for obstetric trauma. Lancet 355(9200), 260-265 (2000).

6 Nyam DC, Pemberton JH. Long-term results of lateral internal sphincterotomy for chronic anal fissure with particular reference to incidence of fecal incontinence. Dis. Colon Rectum 42(10), 1306-1310 (1999).

7 Mitterberger M, Marksteiner R, Margreiter E et al. Myoblast and fibroblast therapy for post-prostatectomy urinary incontinence: 1-year followup of 63 patients. J. Urol. 179(1), 226-231 (2008).

- Myoblasts and fibroblasts implanted via ultrasound-guided transurethral injections is safe and effective therapy for postprostatectomy incontinence, up to 1 year following implantation.

8 Mitterberger M, Pinggera GM, Marksteiner R et al. Adult stem cell therapy of female stress urinary incontinence. Eur. Urol. 53(1), 169-175 (2008).
Centre. The authors have no other relevant affiliations or financial involvement with any organization or entity with a financial interest in or financial conflict with the subject matter or materials discussed in the manuscript apart from those disclosed.

No writing assistance was utilized in the production of this manuscript.

\section{Open access}

This work is licensed under the Creative Commons Attribution 4.0 License. To view a copy of this license, visit http://creativecommons.org/licenses/by/4.0/

- Minimally invasive ultrasound-guided transurethral injections of autologous myoblasts and fibroblasts can cure stress urinary incontinence in women.

9 Frudinger A, Kolle D, Schwaiger W et al. Muscle-derived cell injection to treat anal incontinence due to obstetric trauma: pilot study with 1 year follow-up. Gut 59(1), 55-61 (2010).

- Autologous myoblasts injected into external anal sphincter defects in women presenting fecal incontinence improved continence due to obstetric anal sphincter trauma.

10 Fynes MM, Marshall K, Cassidy M et al. A prospective, randomized study comparing the effect of augmented biofeedback with sensory biofeedback alone on fecal incontinence after obstetric trauma. Dis. Colon Rectum 42(6), 753-758 (1999).

11 Kahyaoglu Sut H, Balkanli Kaplan P. Effect of pelvic floor muscle exercise on pelvic floor muscle activity and voiding functions during pregnancy and the postpartum period. Neurourol. Urodyn. doi:10.1002/nau.22728 (2015) (Epub ahead of print).

12 Pickrell KL, Broadbent TR, Masters FW et al. Construction of a rectal sphincter and restoration of anal continence by transplanting the gracilis muscle; a report of four cases in children. Ann. Surg. 135(6), 853-862 (1952).

13 Christiansen J, Rasmussen OO, Lindorff-Larsen K. Longterm results of artificial anal sphincter implantation for severe anal incontinence. Ann. Surg. 230(1), 45-48 (1999).

14 Lehur PA, Michot F, Denis P et al. Results of artificial sphincter in severe anal incontinence. Report of 14 consecutive implantations. Dis. Colon Rectum 39(12), 1352-1355 (1996).

15 Parker SC, Spencer MP, Madoff RD et al. Artificial bowel sphincter - long-term experience at a single institution. Dis. Colon Rectum 46(6), 722-729 (2003).

16 Baeten CG, Bailey HR, Bakka A et al. Safety and efficacy of dynamic graciloplasty for fecal incontinence: report of a prospective, multicenter trial. Dynamic Graciloplasty Therapy Study Group. Dis. Colon Rectum 43(6), 743-751 (2000)

17 Madoff RD, Rosen HR, Baeten CG et al. Safety and efficacy of dynamic muscle plasty for anal incontinence: lessons from a prospective, multicenter trial. Gastroenterology 116(3), 549-556 (1999). 
18 Boyle DJ, Murphy J, Gooneratne ML et al. Efficacy of sacral nerve stimulation for the treatment of fecal incontinence. Dis. Colon Rectum 54(10), 1271-1278 (2011).

19 Jarrett ME, Varma JS, Duthie GS et al. Sacral nerve stimulation for faecal incontinence in the UK. Br. J. Surg. 91(6), 755-761 (2004).

20 Tjandra JJ, Lim JF, Matzel K. Sacral nerve stimulation: an emerging treatment for faecal incontinence. ANZ J. Surg. 74(12), 1098-1106 (2004).

21 Jiang T, Xia Z, Cheng D et al. Short-term outcomes of adjustable single-incision sling (Ajust ${ }^{\mathrm{TM}}$ ) procedure for stress urinary incontinence: a prospective single-center study. Eur. J. Obstet. Gynecol. Reprod. Biol. 186, 59-62 (2015).

22 Laurikainen E, Valpas A, Kivela A et al. Retropubic compared with transobturator tape placement in treatment of urinary incontinence: a randomized controlled trial. Obstet. Gynecol. 109(1), 4-11 (2007).

23 Fynes M, Donnelly VS, O'Connell PR et al. Cesarean delivery and anal sphincter injury. Obstet. Gynecol. 92(4 Pt 1), 496-500 (1998).
24 Soffer EE, Hull T. Fecal incontinence: a practical approach to evaluation and treatment. Am. J. Gastroenterol. 95(8), 1873-1880 (2000).

25 Frudinger A, Pfeifer J, Paede J et al. Autologous skeletalmuscle-derived cell injection for anal incontinence due to obstetric trauma: a 5-year follow-up of an initial study of 10 patients. Colorect. Dis. 17(9), 794-801 (2015).

-• Autologous skeletal-muscle-derived cells that were used to treat obstetric anal incontinence caused a sustained improvement in incontinence episodes, physiological measurements of anal function, and quality of life at 5 years.

26 Harris SS, Link CL, Tennstedt SL et al. Care seeking and treatment for urinary incontinence in a diverse population. J. Urol. 177(2), 680-684 (2007).

27 Johnson TM, Ouslander JG, Uman GC et al. Urinary incontinence treatment preferences in long-term care. J. Am. Geriatr. Soc. 49(6), 710-718 (2001).

28 Kim HL, Gerber GS, Patel RV et al. Practice patterns in the treatment of female urinary incontinence: a postal and internet survey. Urology 57(1), 45-48 (2001). 